\title{
Radiosynoviorthesis for Treatment of Hemophilic Hemarthrosis-Slovenian Experience
}

\author{
Marko Grmek, ${ }^{1}$ Metka Milčinski, ${ }^{1}$ Jurij Fettich, ${ }^{1}$ Majda Benedik-Dolničar, ${ }^{2}$ and Janez Brecelj ${ }^{3}$ \\ ${ }^{1}$ Department for Nuclear Medicine, ${ }^{2}$ Children's Hospital Oncology-Hematology Unit, and ${ }^{3}$ Department \\ for Orthopedic Surgery, University Medical Center Ljubljana, Ljubljana, Slovenia.
}

\begin{abstract}
Radiosynoviorthesis is a well-accepted method for the treatment of recurrent hemarthrosis in hemophilic patients. Objectives: The aims of our study were to evaluate the effectiveness of radiosynoviorthesis in patients suffering from hemophilic hemarthrosis, to determine the effect of treatment on antihemophilic factor consumption, and to assess the patient's satisfaction with radiosynoviorthesis. Methods: Between 2001 and 2003, 26 radiosynoviortheses were done in 21 hemophilic patients; in 4 patients the treatment was repeated, and in 1 patient two joints were treated. ${ }^{90} Y$ colloid was used for the knee joint, and ${ }^{186} R e$ colloid was used for ankle, shoulder, and elbow radiosynoviorthesis. Results: The bleeding frequency decreased by at least $50 \%$ in $53 \%$ of patients in the year after radiosynoviorthesis, as compared to the year prior to the therapy. Considering only those patients who had at least 12 bleedings into the treated joints in the year preceding the therapy, the bleeding frequency decreased by at least $50 \%$ in $62 \%$ of these patients. In this group, the consumption of the antihemophilic factor was notably reduced (on average, by 25,800 I.U./year). All patients reported that the treated joint was much better or better than before the radiosynoviorthesis. Conclusion: Radiosynoviorthesis is an effective method for the treatment of hemophilic hemarthrosis, particularly in patients with frequent intra-articular bleedings. The antihemophilic factor consumption was markedly reduced only in patients with frequent joint bleeding. Radiosynoviorthesis is well accepted by patients suffering from hemophilic hemarthrosis.
\end{abstract}

Key words: hemophilia, radiosynoviorthesis

\section{INTRODUCTION}

In patients with severe hemophilia, serious bleeding can occur spontaneously. A total of $85 \%$ of all bleeding events occur in joints, and $80 \%$ of such events affect the knee, ankle, and elbow, with the hip and shoulder being involved to a

Address reprint requests to: Marko Grmek; Department for Nuclear Medicine, University Medical Center Ljubljana; Ljubljana, Slovenia; Tel.: ++386-1-522-3488; Fax: $++386-1-432-7727$

E-mail: marko.grmek@guest.arnes.si lesser extent. ${ }^{1}$ Acute hemarthrosis is a consequence of rapid articular bleeding, and is characterized by rapid swelling of the joint. ${ }^{2}$ It may be preceded by a prodrome of stiffness and pain. Patients with recurrent articular bleeding, especially those with severe hemophilia, develop chronic hemarthrosis with synovitis and progressive arthropathy. Different therapeutic options are available to prevent the development of hemophilic arthropathy, one being radiosynoviorthesis. ${ }^{3-5}$

Radiosynoviorthesis (radiosynovectomy) is a method of treatment of joint synovitis or synovial 
process by intra-articular injection of a $\beta$-emitting radionuclide. ${ }^{6}$ Radiation from radioactive isotope causes fibrosis in the subsynovial connective tissue of the joint capsule and synovial villi. It also affects the complex vascular system of the synovial membrane, resulting in the closure of some vessels. ${ }^{7}$ On this basis, a self-perpetuating cycle of hemarthrosis-synovitis-hemarthrosis can be stopped and joint degeneration prevented. The ideal candidate for radiosynoviorthesis is a hemophilic patient with recurrent hemarthrosis (two to three bleeds per month) in a target joint without radiological changes, in whom conservative treatment with antihemophilic factor (AHF) replacement and physiotherapy has failed. ${ }^{2}$ Such patients, however, are rarely encountered in practice. As reported in the literature, radiosynoviorthesis was also used in patients who did not meet all the above-mentioned criteria. ${ }^{2}$ The aim of our study was to evaluate the effectiveness of radiosynoviorthesis in patients with hemophilic hemarthrosis, as well as to determine its effect on the AHF consumption, and to assess patients' experience with radiosynoviorthesis.

\section{MATERIALS AND METHODS}

\section{Patients}

In Slovenia, the majority of hemophilic patients are self-treated with AHF in accordance with a hematologist's recommendation. All hemophilic patients and medical records concerning their treatment and site of bleeding are kept in the central hemophilia registry.

Between January 2001 and December 2003, 26 radiosynoviorthesis were performed in 21 hemophilic patients in the University Medical Center Ljubljana, Slovenia. Nineteen patients had hemophilia A, 1 patient hemophilia B, and 1 female patient had von Willebrand disease. The patients were from 11 to 54 years of age (median, 24 years). In total, 22 joints ( 8 elbows, 6 knees, 1 shoulder, and 7 ankle joints) were treated, because 1 patient underwent radiosynoviorthesis for hemarthrosis involving two joints. Four patients were treated with repeated radiosynoviorthesis.

An experienced orthopedic surgeon examined hemophilic patients with an increased rate of articular bleedings. Indication for radiosynoviorthesis was given on the grounds of the joint bleeding history, clinical picture, and morphological findings on radiogram. Before radiosyn- oviorthesis, the patients received a detailed explanation of the expected effects and possible complications of the procedure, and gave written consent to the therapy. They were injected with the AHF in a dose determined by the hematologist prior to the radiosynoviorthesis.

\section{Methods}

Administration of the radioactive colloid into the joint was performed as follows: the patient was placed in an appropriate position by the orthopedic surgeon, who determined and prepared the application site. After local infiltration with $1 \%$ xylocaine, a 21-gauge needle was inserted into the joint. In knee radiosynoviorthesis, the intra-articular position of the needle was confirmed by aspiration of synovial fluid. In other joints, the intra-articular position of the needle tip was controlled by X-ray screening after an injection of radiographic contrast. Once the needles' position inside the joint was confirmed, a radioactive isotope was injected into the joint. In 3 patients presenting with evident synovitis, a long-acting glucocorticoid was injected into the joint. In other patients, the needle was flushed with a small volume of saline before being withdrawn from the articular space. After removal of the needle, the joint was sensibly moved and the extremity was immobilized in a dorsal plaster splint for 4 days.

The following radiopharmaceuticals recommended by the European Association of Nuclear Medicine (EANM) were used: ${ }^{90} \mathrm{Y}$ silicate, activity $185 \mathrm{MBq}$, for knee radiosynoviorthesis and ${ }^{186} \mathrm{Re}$ sulfide, activities $111 \mathrm{MBq}$ for the shoulder, $74 \mathrm{MBq}$ for the elbow, and $74 \mathrm{MBq}$ for the ankle radiosynoviorthesis. ${ }^{6}$ In patients treated with ${ }^{186} \mathrm{Re}$ colloid, the affected joint, regional lymph nodes, kidneys, and bladder were evaluated with scintigraphy 24 hours after the procedure. Distribution of ${ }^{186} \mathrm{Re}$ in the ankle joint after radiosynoviorthesis is shown in Figure 1.

One year after the procedure, the patients gave answers to the following questions:

- Is the treated joint worse, the same, better, or much better than before the radiosynoviorthesis?

- Did you have any adverse reactions after the application of the radiopharmaceutical?

- If needed, would you decide for radiosynoviorthesis again?

The effectiveness of radiosynoviorthesis was evaluated on the basis of the number of bleeding 
Table 1. Outcomes of Radiosynoviorthesis in Joints with $<12$ Bleedings, and Joints with $\geq 12$ Bleedings, Compared to the Year Preceding the Procedure ${ }^{\mathrm{a}}$

Number of bleedings into the treated joint

in the year preceding radiosynoviorthesis

Number of treated joints

Average number of bleedings into the treated joint

in the year:

Before treatment (mean \pm SD)

(median)

After treatment (mean \pm SD)

(median)

Outcome of the treatment:

\section{Excellent}

Good

Moderate

Absent

Average consumption of AHF (I.U.) used for control

of bleeding into the treated joint in the year:

Before treatment (mean $\pm \mathrm{SD}$ )

(median)

After treatment (mean \pm SD)

(median)

Mean difference

aThe treatment success and saving in AHF consumption are given.

AHF, antihemophilic factor; I.U., international units; SD, standard deviation.

Outcome of theory: excellent ( $\geq 90 \%$ reduction of hemarthrosis); good (decrease by $50 \%-89 \%$ ); moderate (decrease by $10 \%-49 \%$ ); and absent (decrease by $<10 \%$ ).

episodes and the amount of the AHF consumed prior to the procedure and during the postradiosynoviorthesis period. The central hemophilia registry provided the data. The outcomes of the therapy were rated as:

- Excellent: The number of articular bleeding episodes decreased by at least $90 \%$;

- Good: The number of hemarthrosis declined by 50\%-89\%;

- Moderate: The number of hemarthosis decreased by $10 \%-49 \%$;
- Absent: The number of bleedings decreased by less than $10 \%$, or increased.

\section{Statistical methods}

Descriptive statistical methods were used for data and results presentation.

\section{RESULTS}

One year after radiosynoviorthesis, the results of the procedure could be evaluated for 19 joints in

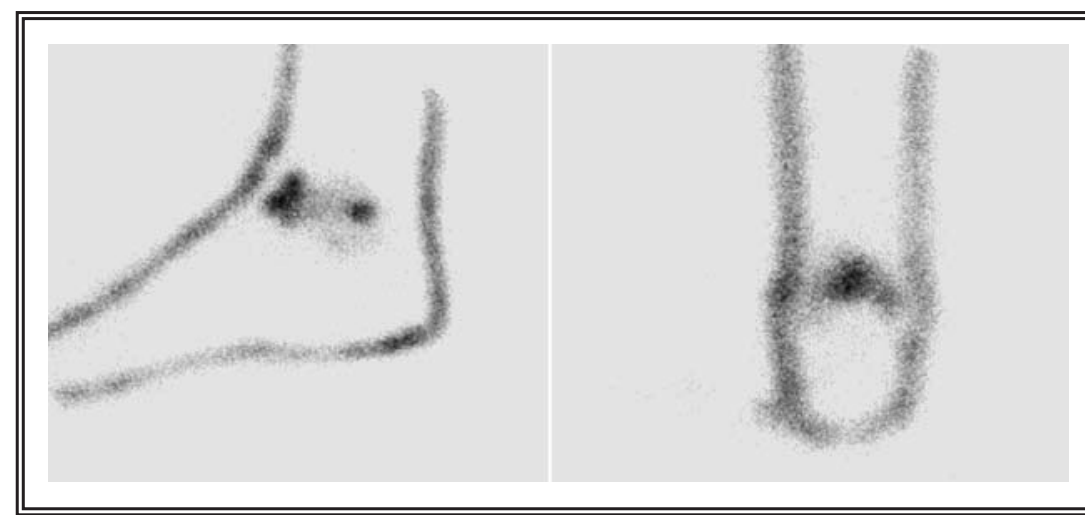

Figure 1. A scintigram taken 24 hours after radiosynoviorthesis: distribution of ${ }^{186} \mathrm{Re}$ colloid in the ankle joint is shown. The foot is outlined with additional radioactive source. 
Table 2. Outcomes of Radiosynoviorthesis in the 1st-, 2nd-, 3rd-, and 4th 6-Month Period After the First Treatment Given in Number of Joints and the Percentage (\%).

\begin{tabular}{|c|c|c|c|c|c|}
\hline \multirow{2}{*}{$\begin{array}{l}\text { Time after } \\
\text { radiosynoviorthesis } \\
\text { (months) }\end{array}$} & \multirow{2}{*}{$\begin{array}{l}\text { Number of } \\
\text { treated } \\
\text { joints }\end{array}$} & \multicolumn{4}{|c|}{ Outcome of the treatment } \\
\hline & & Excellent & Good & Moderate & Absent \\
\hline $1-6$ & 22 & $5(23 \%)$ & $8(36 \%)$ & $5(23 \%)$ & $4(18 \%)$ \\
\hline $7-12$ & 17 & $6(35 \%)$ & $2(12 \%)$ & $3(18 \%)$ & $6(35 \%)$ \\
\hline $13-18$ & 14 & $3(21 \%)$ & $5(36 \%)$ & 0 & $6(43 \%)$ \\
\hline $19-24$ & 8 & $1(13 \%)$ & $1(13 \%)$ & 0 & $6(74 \%)$ \\
\hline
\end{tabular}

Outcome of therapy: excellent ( $\geq 90 \%$ reduction of hemarthrosis), good (decrease by $50 \%-89 \%$ ), moderate (decrease by $10 \%-49 \%)$, and absent (decrease by $<10 \%$ ).

17 patients. During the year preceding the therapy, the patients reported 3-30 spontaneous bleedings into the joint treated by radiosynoviorthesis. The effect of the treatment and the decrease in the AHF consumption, compared to the situation in the year preceding the procedure, are indicated in Table 1. The effectiveness of radiosynoviorthesis in joints with less than 12 bleedings $(<12)$, and joints with 12 or more bleedings $(\geq 12)$ are presented separately.

The outcome of treatment was followed for at least 6 months in 22 joints after the first radiosynoviorthesis. The effectiveness of treatment during 6-month periods after the therapy, as compared to the six-month average in the year preceding the therapy, is presented in detail in Table 2.

The effectivenes of knee, ankle, elbow, and shoulder radiosynoviorthesis evaluated as a decrease of bleeding frequency in the 1st year after treatment is presented in Table 3.

In four joints, recurrent hemarthrosis or poor effect of the first therapy required repeat ra- diosynoviorthesis, on average, 1 year and 5 months after the first treatment. The outcomes of retreatment were: good in one joint, moderate in two joints, and absent in one joint.

Subjective replies of hemophilic patients, who were treated by radiosynoviorthesis, to the questionnaire were as follows:

- $55 \%$ of patients answered that the treated joint was much better than before, and $45 \%$ of patients said that the joint was better than before.

- After the treatment, $55 \%$ of patients had no adverse reactions; $37 \%$ had mild transitory swelling, and $7 \%$ developed moderate transitory swelling of the treated joint.

- All patients would decide for radiosynoviorthesis again.

\section{DISCUSSION}

In the University Medical Centre Ljubljana, Slovenia radiosynoviorthesis is routinely used

Table 3. Outcomes of Knee, Ankle, Elbow and Shoulder Radiosynoviorthesis in the First Year Following Therapy-in the First (1-6) and Second (7-12) 6-Month Period

\begin{tabular}{|c|c|c|c|c|c|c|}
\hline \multirow{2}{*}{$\begin{array}{l}\text { Treated } \\
\text { joint }\end{array}$} & \multirow{2}{*}{$\begin{array}{c}\text { Time after } \\
\text { radiosynoviorthesis } \\
\text { (month) }\end{array}$} & \multirow{2}{*}{$\begin{array}{c}\text { Number of } \\
\text { treated } \\
\text { joints }\end{array}$} & \multicolumn{4}{|c|}{ Outcome of the treatment } \\
\hline & & & Excellent & Good & Moderate & Absent \\
\hline \multirow[t]{2}{*}{ Knee } & $1-6$ & 6 & 1 & 1 & 2 & 2 \\
\hline & $7-12$ & 6 & 1 & 0 & 1 & 4 \\
\hline \multirow[t]{2}{*}{ Ankle } & $1-6$ & 7 & 1 & 2 & 2 & 2 \\
\hline & $7-12$ & 5 & 2 & 0 & 2 & 1 \\
\hline \multirow[t]{2}{*}{ Elbow } & $1-6$ & 8 & 3 & 4 & 1 & 0 \\
\hline & $7-12$ & 5 & 3 & 2 & 0 & 0 \\
\hline \multirow[t]{2}{*}{ Shoulder } & $1-6$ & 1 & 0 & 1 & 0 & 0 \\
\hline & $7-12$ & 1 & 0 & 0 & 0 & 1 \\
\hline
\end{tabular}

Outcome of therapy: excellent ( $\geq 90 \%$ reduction of hemarthrosis), good (decrease by $50 \%-89 \%$ ), moderate (decrease by $10 \%-49 \%$ ), and absent (decrease by $<10 \%$ ). 
more than 15 years for the treatment of rheumatic synovitis, and in 2001, it has been introduced for hemophilic patient. The introduction of the radiosynoviorthesis in the clinical practice in Slovenia (population of 2 million) was a joint effort of an experienced orthopedic surgeon and of a specialist of nuclear medicine. An average of 9 hemophilic patients were treated every year.

The outcomes obtained in our series were slightly worse than those reported in the literature, most likely owing to the inclusion of a greater number of patients with a relatively low rate of bleedings into the treated joint in our study. ${ }^{8-13}$ In patients who suffered 12 or more bleedings into the treated joint in the year preceding the treatment, the outcomes are practically the same as those reported in the literature. ${ }^{8-13}$ The probability for excellent or good results was $62 \%$, and the average AHF consumption was decreased by $60 \%$ in this subgroup. Treatment of joints with less than 12 hemarthrosis apparently yielded results that were not so good. Although the therapy produced no significant decline in the number of hemarthrosis, the results of radiosynoviorthesis were judged fair in $45 \%$ of patients. On detailed data examination, 4 patients stood out of this subgroup. In 2 of the patients, the bleeding frequency increased in the last 3 months before radiosynoviorthesis (11 and 8 bleeds), and in both patients the bleeding frequency increased in the year after treatment (23 and 17 bleeds, respectively). Two other patients had a history of only three intra-articular bleedings and severe hypertrophic synovitis 1 year preceding therapy. After the treatment, the number of hemarthrosis remained unchanged, but the treated joints improved in both cases. No uniform recommendations defining the criteria for radiosynoviorthesis had been published yet. Regarding the number of hemarthrosis before treatment, further studies on a greater number of patients should be conducted, with the aim for a better determination of appropriate candidates.

The effect of radiosynoviorthesis declined with time. The exception was the 2nd 6-month period after the procedure, when even a smaller number of bleeding episodes were reported than in the preceding 6 months. This finding was most probably the consequence of a delay in fibrotic process after ionizing radiation. Time-dependent decreasing effect of treatment was comparable to those reported in the literature. ${ }^{14}$

The best outcome of treatment was obtained in hemophilic patients experiencing bleedings into the elbow joint, good results were recorded for treatment of ankle hemarthrosis, and moderate results were yielded by knee joint radiosynoviorthesis. The elbow and the ankle joints were treated with ${ }^{186} \mathrm{Re}$ colloid and the knee joint with ${ }^{90} \mathrm{Y}$ colloid. The differences in the effectiveness of radiosynoviorthesis between the treated joints, however, do not seem to be a result of the use of different radiopharmaceutcals. Our results resemble those from the literature. ${ }^{9}$

As $44 \%$ of the patients included in the survey reported mild transitory swelling of the treated joint, simultaneous long-acting glucocorticoid administration is suggested.

\section{CONCLUSION}

Radiosynoviorthesis is an effective method for the treatment of hemophilic hemarthrosis, particularly in patients experiencing frequent bleeding episodes. In joints with 12 or more bleedings per year, a marked reduction of antihemophilic factor consumption was observed.

Radiosynoviorthesis is well accepted by hemophilic patients suffering from hemophilic hemarthrosis.

\section{REFERENCES}

1. Roosendaal G, Van den Berg HM, Lafeber FPJG, et al. Blood-induced joint damage: An overview of musculoskeletal research in haemophilia. In: Rodriguez-Merchan EC, Goddard NJ, Lee CA, eds. Musculoskeletal Aspects of Haemophilia. Oxford: Blackwell Science, 2000:18.

2. Silva M, Luck JV, Jr, Llinás A. Chronic hemophilic synovitis: The role of radiosynovectomy. Montréal: World Federation of Hemophilia, 2004.

3. Hilgartner MW. Current treatment of hemophilic arthropathy. Curr Opin Pediatr 2002;14:46.

4. Muller S, Kurth AA, Hovy L. Conservative treatment measures in hemophilic arthropathy [in German]. Orthopaede 1999;28:347.

5. Rodriguez-Merchan EC. Methods to treat chronic haemophilic synovitis. Haemophilia 2001;7:1.

6. EANM Procedure guidelines for radiosynovectomy. Available at: http:/www.eamn.org/scientific_info/ guide_html/gl_synovectomy.html Accessed on July 19, 2004.

7. Rodriquez-Merchan EC, Goddard NJ. Chronic haemophilic synovitis. In: Rodriguez-Merchan EC, Goddard NJ, Lee CA, eds. Musculoskeletal Aspects of Haemophilia. Oxford: Blackwell Science, 2000:43. 
8. Siegel HJ, Luck JV, Jr, Siegel ME, et al. Phosphate-32 colloid radiosynovectomy in hemophilia: Outcome of 125 procedures. Clin Orthop 2001;392:409.

9. Rodriguez-Merchan EC, Jimenez-Yuste V, Villar A, et al. Yttrium-90 synoviorthesis for chronic haemophilic synovitis: Madrid experience. Haemophilia 2001;7 (Suppl 2):34.

10. Fernandez-Palazzi F, Rivas S, Cibeira JL, et al. Radioactive synoviorthesis in hemophilic hemarthrosis: Materials, techniques, and dangers. Clin Orthop 1996;328:14.

11. Heim M, Goshen E, Amit Y, et al. Synoviorthesis with radioactive yttrium in haemophilia: Israel experience. Haemophilia 2001;7(Suppl 2):36.

12. Fernandez-Palazzi F. Treatment of acute and chronic synovitis by nonsurgical means. Haemophilia 1998; 4:518.

13. Kresnik E, Mikosch P, Gallowitsch HJ, et al. Clinical outcome of radiosynoviorthesis: A meta-analysis including 2190 treated joints. Nucl Med Comm 2002; 23:683.

14. Silva M, Luck JV, Jr, Siegel ME. 32 P chromic phosphate radiosynovectomy for chronic haemophilic synovitis. Haemophilia 2001;7(Suppl 2):40. 